\title{
An Industrial Big Data Processing Engine
}

\author{
Aguinaldo Bezerra* Vitor Greati ${ }^{* *}$ Vinícius Ribeiro** \\ Ivanovitch Silva* Luiz Affonso Guedes* \\ * Programa de Pós-Graduação em Engenharia Elétrica e de \\ Computação, Universidade Federal do Rio Grande do Norte, Natal, \\ RN,Brazil,(e-mails: aguinaldo@ufrn.edu.br,ivan@imd.ufrn.br, \\ affonso@dca.ufrn.br). \\ ** Departamento de Informática e Matemática Aplicada, Universidade \\ Federal do Rio Grande do Norte, Natal, RN, Brazil (e-mails: \\ greati@ufrn.edu.br,vinihcampos@ufrn.edu.br)
}

\begin{abstract}
Industries are generally data rich but information poor environments. Massive data generated in industrial operations is traditionally neglected (or simply took aside) mainly due to systems design restrictions, to the lack of adequate processing power of typically installed computing infrastructure and to a sector culture notably focused on collecting, selecting, storing and preserving historical series in on-demand access repositories. This huge amount of unprocessed data resting in these repositories is a latent source of information that could be used to improve industrial processes. This work then proposes an approach in which a proper computing power processing engine is plugged-in to current industrial information infrastructure to provide it with the ability of handling massive industrial data. Testing on real-world industrial data volumes of $5 \mathrm{~GB}, 50 \mathrm{~GB}$ and $100 \mathrm{~GB}$ attested the effectiveness and potential of the proposed approach in dealing with Industrial Big Data.
\end{abstract}

Keywords: Industrial Big Data; Information Infrastructure; Parallel Processing; Dask; Hadoop; Sqoop.

\section{INTRODUCTION}

Constant growth, technological advances and paradigm shifts, innate to industry, have pushing the sector towards the undeniable reality of data orientation. Current trends in modern manufacturing point towards processing its data into timely and valuable information to support decision making and the continuous improvement of industrial processes. In this context, Industry 4.0 emerges as a watershed in manufacturing, from the synergy of the availability of innovative digital technology and the demand by consumers for high quality and customized products (Kagermann et al., 2013; Silva et al., 2018). Referring to the so-called $4^{\text {th }}$ industrial revolution, Industry 4.0 is an umbrella term which outlines a series of paradigm shifts by which traditional industries have been going through to ensure survival in global scenario of high competitiveness (Bartodziej, 2017; Kagermann et al., 2013). Although the term is defined from various and diverse perspectives, a point of convergence is that Industry 4.0 represents a revolution towards digitization and computerization of manufacturing which is transforming production and management ( $\mathrm{Lu}, 2017$; Bartodziej, 2017).

Under that umbrella, interrelated concepts such as CyberPhysical Systems (CPS), Smart Factory (SF), (Industrial) Internet of Things (I-IoT) are perceived as key components and have been receiving great appeal. In that respect, as a natural and direct consequence of these paradigm shifts, volume, velocity and variety of data industries have to manage is exploding at really high rates (GE Intelligent
Platforms, 2012; Obitko et al., 2013), and, consequently, an infrastructure capable of embracing this mass of data is needed to be established. To cope with that, some enabling technologies related to the concept of Big Data, which have been typically and solidly been employed in the Information Techology (IT) domain, have been also applied to meet industrial reality and needs (Gokalp et al., 2016). However, direct and large-scale application of mainstream big data tools and methods is not a trivial task, due to domain-specific challenges such as diverse communication standards, proprietary information and automation systems, heterogeneous data structures and interfaces and inflexible governance policies, coupled with the lack of inherent support from those tools and methods to industrial applications (Gokalp et al., 2016; O'Donovan et al., 2015). In this perspective, recent literature have come on the subject of Industrial Big Data with a particular regard to problems and challenges to be overcome in face of the indefeasible absorption of this new paradigm in automation. Proposals of suitable Big Data approaches and architectures to meet industrial needs (Lee et al., 2014; Gokalp et al., 2016; Mourtzis et al., 2016; Basanta-Val, 2018; Lee et al., 2015; Wan et al., 2017) suggests Industrial Big Data is still a developing field of study.

This work then makes a brief review regarding the subject Industrial Big Data and its related infrastructure and introduces an approach in which mainstream open-source tools are used to setup IBiDaPE (Industrial Big Data Processing Engine), an engine meant to be plugged-in to conventionally installed industrial infrastructures, provi- 
ding them with the ability to perform Big Data analysis over voluminous industrial data repositories.

The remainder of the paper is structured as follows. In Section 2, paper background subject of industrial Big Data infrastructure is discussed. Section 3 introduces our approach to cope with massive industrial data. In Section 4 , a case study with some test scenarios and their respective results are presented. Finally, Section 5 concludes the paper and envisions future directions.

\section{INDUSTRIAL BIG DATA INFRASTRUCTURE}

As traditional "small data" computational approaches are proven to be ineffective in dealing with a high magnitude data volume (Philip Chen and Zhang, 2014), new coping approaches and a differentiated computational infrastructures are demanded. This refers basically to the ability of properly handling capture, storage, management, querying, share and visualization of this huge amount of data in this increasing data magnitude and importance scenario. This aptitude is made possible through the advent of new computing paradigms such as cloud computing, jungle computing and fog computing, which are elementally based on distributed, parallel and high performance computing (Hajibaba and Gorgin, 2014).

Data-driven approaches have been supporting decisionmaking processes over the last years in a myriad of sectors and this was enabled by technologies pioneered in the IT realm (Jeble et al., 2018). Not unlike, data-driveness has also made its path through industry. It happened in a natural way, once manufacturing generates and stores more data than any other sector (Brian Hartmann and Narayanan, 2015; Obitko et al., 2013) and given the not so new industry convergence to IT-bond technologies.

Current industrial computing infrastructure, in terms of management and processing of plant-related data, is mainly focused on collecting, selecting and storing data at appropriate rates, preserving historical series in ondemand access repositories (Obitko et al., 2013). Mostly due to a design restriction, any additional processing such as deeper queries or analysis are beyond the capacity of typically installed computing infrastructure. Thus, a current trend in industrial systems refers to the use of different Big Data precepts as a mean to process this huge and dormant amount of data generated in industrial plants which cannot be processed with a conventional infrastructure. This is a base and necessary step to put industry on track of the crescent data inundation scenario.

Industrial Big Data is an already well known concept which refers essentially to the absorption of Big Data in Industry. As surveyed in Basanta-Val (2018), it inherits the defining characteristics of general purpose Big Data concept such as volume, variety, velocity, variability and veracity ( $5 \mathrm{Vs})$, as well as extends this concept by adding two new Vs: visibility, which regards to the discovery of unexpected insights of existing processed data; and value, which puts emphasis on creating new value from massive data. In that work is also stated that industrial data is more structured, correlated and ready to analysis since it is generated by automated equipment in more controlled environments and processes.
Perform collection, aggregation, handling and processing of the real data mass of data is indeed a challenge to be overcome. To make most data-driven approaches feasible and viable in such a voluminous data and timerestricted scenario, it is necessary to establish a hardware and software infrastructure capable of meeting the great computational demands of those approaches.

Industry has been aligning to some Big Data precepts to accomplish such a kind of processing which, in terms of computational infrastructure, includes, among other aspects, a high computing power, parallel, distributed and online processing of massive data capabilities, distributed file systems with safe access, intelligent, fast and robust communications infrastructure, as well as an analysis systematization based on the specific plant knowledge (Obitko et al., 2013; Basanta-Val, 2018; Lee et al., 2015).

Literature confirms this matter as a high relevance interest topic. A recent contribution concerns the establishment of a Big Data engine able of meeting industrial needs and peculiarities (Basanta-Val, 2018). In that work, the author permeates the potential of a collection of Big Data architectures, optimization approaches and Big Data benchmarking tools in industry and introduces a conception of an industrial Big Data architecture capable of addressing the limitations and improve efficiency of those architectures in relation to their map-reduce models. Another promising reference in this area defines a cloud-assisted architectures, and mechanisms for Big Data data processing in the field of preventive maintenance are proposed and compared (Wan et al., 2017). Other prominent contribution is a survey focused on key techniques for designing and implementing efficient and high performance industrial Big Data analytics platforms (Wang et al., 2018). This recurrence proves that the establishment of an Industrial Big Data infrastructure figures as a requirement for today's industries.

\section{INDUSTRIAL BIG DATA PROCESSING ENGINE}

Industry is an inherently conservative sector with respect to its policies, methods and procedures. Thus, besides being a current top-priority topic for most industries, the pursuit for Industry 4.0 is a progressive and forwardlooking continuous improvement process that has just started and has a long way ahead. A plausible move in this gradual approach to Industry 4.0 is to improve operational performance and governance by proactively and timely exploring the great amount of potential valuable information hidden in massive industrial data repositories such as loggers and historians. To make it feasible, this work proposes the establishment of a minimum working Big Data engine which is capable of dealing with those voluminous data repositories without interfering on or impacting current installed information infrastructure. This engine, named Industrial Big Data Processing Engine (IBiDaPE), is meant to be attached as a processing engine to industrial data repositories, normally located at the management/planning layer (composed mainly by Process Information Management Systems - PIMS and Manufacturing Execution Systems - MES) layer on the automation pyramid, to provide this layer with the ability 
of processing those large amounts of data and extract value from previously neglected data assets. Figure 1 outlines the role of IBiDaPE in the functional scope of the classical automation pyramid.

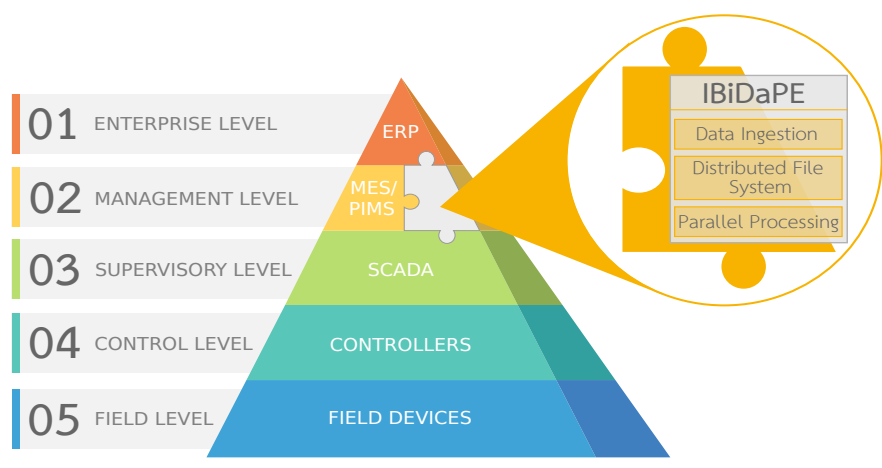

Figura 1. IBiDaPE role in the automation pyramid.

IBiDaPE is built on top of mainstream open-source software solutions. An open-source environment was highly preferred over proprietary solutions to setup this engine mainly due to gains in flexibility, scalability and customization obtained with this choice, as well as due to the abundant documentation, fast-growing community of users and an increasing improvement of these tools, allied with a zero-cost tool-set setup. This constitutes an inexpensive but powerful environment suited to perform diverse and complex Big Data analysis tasks.

IBiDaPE is structured in three layers (illustrated in Figure 2):

- Data Ingestion Layer: It is the bridge between current installed information infrastructure and IBiDaPE. It gathers continuously stored data from SQLbased industrial data repositories such as historians and loggers (data sources) to make it available in the distributed file system of the engine. It uses Sqoop (Apache Software Foundation, 2019) as ingestion tool.

- Data Storage Layer: This layer uses a distributed file system to speed up file system jobs over massive volumes of data. It relies on Hadoop Distributed File System (HDFS) (Shvachko et al., 2010).

- Data Processing Layer: On top of a distributed file system, this layer implements a parallel processing cluster to speed up computation. It counts on Python language library Dask (Dask Development Team, 2016).

Sqoop is the core tool in Data Ingestion Layer. It is a toolset designed to automate the process of accomplishing bi-directional data transfer between HDFS and relational

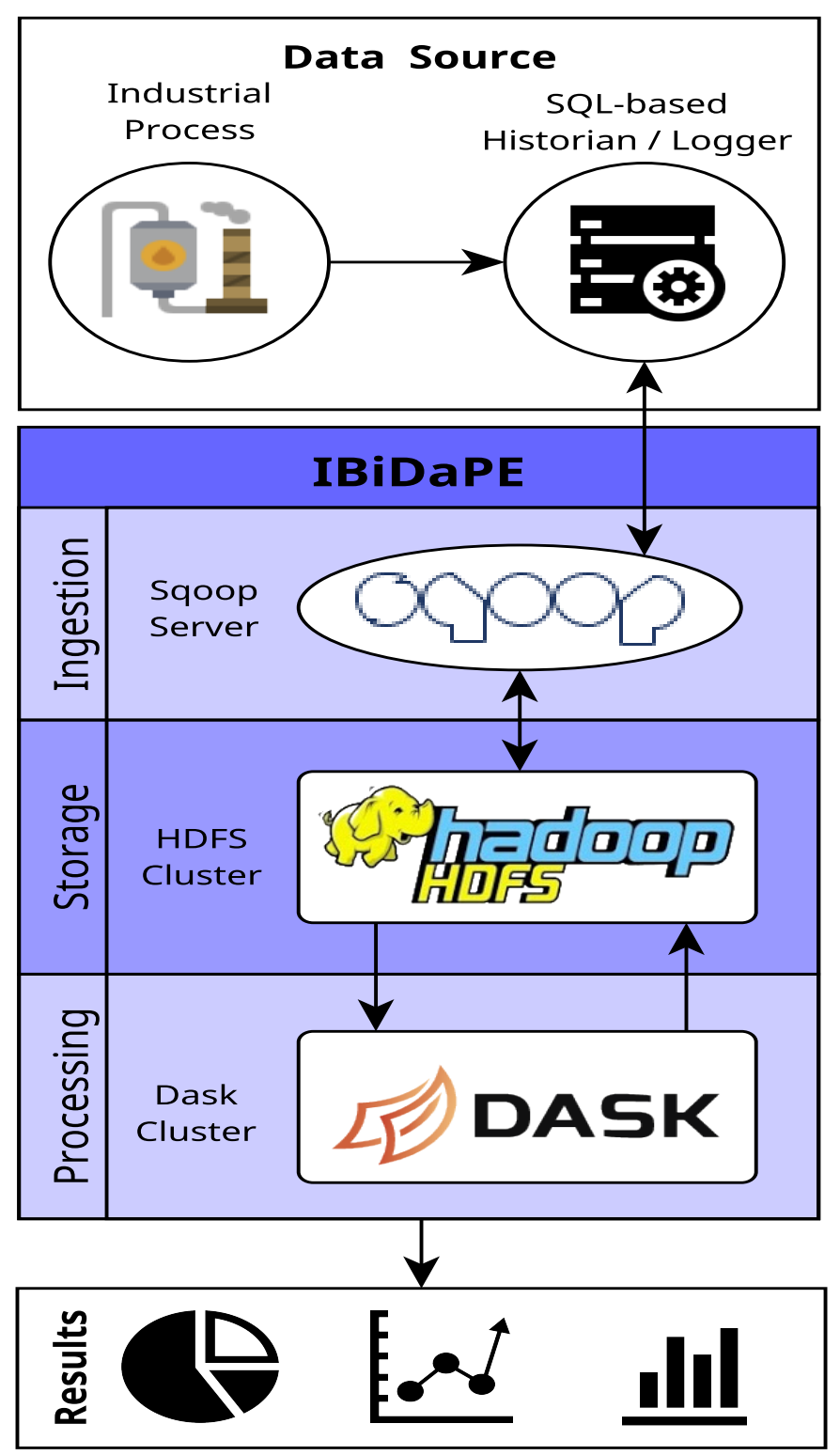

Figura 2. IBiDaPE architecture.

databases (Apache Software Foundation, 2019). The role of Sqoop in this layer is to asynchronously gather large data volumes from SQL-based loggers and historians servers, making them available through a fast and reliable distributed file system, which in our case is HDFS. Data transformations performed over HDFS can be exported back to those loggers and historians. To the moment, Sqoop is the only prodution-ready tool to implement this bridge between SQL and HDFS.

Tabela 1. Sample entries of data set under analysis.

\begin{tabular}{|c|c|c|c|c|c|c|c|}
\hline Date_Time & Type $^{\mathbf{a}}$ & Category $^{a}$ & Module $^{b}$ & State $^{a}$ & Level $^{\mathrm{a}}$ & $\operatorname{Desc1}^{c}$ & $\ldots$ registry_id ${ }^{d}$ \\
\hline 19-02-21 14:16:04 & EVENT & PROCESS & MDL-015783HIC & INACT/ACK & $\mathrm{NaN}$ & RATE & 18794 \\
\hline $19-02-23$ 08:45:36 & EVENT & PROCESS & MDL-414572PID & ACTIVE & INFO & ERROR CLEAR & ... 12946 \\
\hline $19-02-22 \quad 12: 21: 32$ & ALARM & $\mathrm{NaN}$ & MDL-45420VEC & $\mathrm{ACT} / \mathrm{UNACK}$ & ADVISORY & COMM FAIL & ... 5948 \\
\hline $19-02-23$ 15:38:42 & ALARM & PROCESS & MDL-1557452DI & $\mathrm{ACT} / \mathrm{UNACK}$ & CRITICAL & HIHI & ... 6397 \\
\hline $19-02-22 \quad 16: 41: 19$ & EVENT & INSTRUM & MDL-1029ASMB & INACT/ACK & $\mathrm{NaN}$ & MODBAD & $\ldots 24883$ \\
\hline
\end{tabular}

${ }^{\mathrm{a}}$ Registry attributes; ${ }^{\mathrm{b}}$ Registry entities; ${ }^{\mathrm{c}}$ Registry description; ${ }^{\mathrm{d}}$ Registry unique identification. 
HDFS is a wide adoption distributed file system that met the requirements established for the Data Storage Layer of IBiDaPE. This layer depends on a fast and reliable data storage system capable of handling voluminous data supporting replication and redundancy for fault tolerance. HDFS provides a cost-effective and reliable storage capability through a cluster which can handle huge volumes of both structured and unstructured data (Oussous et al., 2017). It is designed for high-latency batch processing operations and has great portability and scalability across heterogeneous hardware and software platforms (commodity nodes) (Dhruba Borthakur, 2019). It uses MapReduce (Dean and Ghemawat, 2004) programming model in file system operations to ensure effective and timely access to data, reducing network congestion and increasing system performance by moving computations near to data storage (Oussous et al., 2017). HDFS then provides redundant, fault tolerant and fast data access for the Data Processing Layer of IBiDaPE.

Data Processing Layer is responsible for performing computations over massive data volumes and is built on top of Dask, a flexible parallel computing library fully implemented in Python language, suited to solve a wide variety of large data handling and analysis problems in scientific computing and general-purpose distributed computing. It natively can scale other well-established Python libraries which comprise the Python Open Data Science Stack (PODSS) such as NumPy, Pandas, and Scikit-Learn (Daniel, 2018) to allow an easy switching from single machine computing power to cluster computing power as data size scales. In Dask, directed acyclic graphs (DAG) are used to compose, control and express computations, which can then be run using a variety of schedulers, from a single machine scheduler using threads, to a distributed scheduler that scales across a cluster (Daniel, 2018). Dask provides parallel versions of familiar Python single-threaded data collections such as NumPy arrays and Pandas dataframes, making transitioning to parallel processing easier (Crist, 2016). A Dask cluster is then set up to provide IBiDaPE with a powerful and easy to use parallel processing ability.

\section{CASE STUDY}

\subsection{Target Data}

Data source in this case study is a table from a relational database of alarm management log server from a petrochemical plant. Database entries refer to time-stamped observations of 28 numeric and categorical variables for relevant plant entities such as sensors, actuators and controllers. Table 1 shows some sample entries of the data set in tabular format. In this table, some columns were omitted for the sake of limited horizontal space and data is sanitized to hide sensitive information.

\subsection{Test Scenarios}

Targeting that data source, a comparative three-scenario case study is set up:

- Scenario 1: Operations were performed directly over the SQL-based alarm management log server using SQL queries through the default Database Management Systems (DBMS) client. DBMS was used in its standard settings and cursors were used to handle data volumes that do not fit in memory.

- Scenario 2: In disk SQL dumps (retrieved with the default DBMS client) referring to data volumes were handled on a standard workstation using PODSS. Chunking was used to manipulate data volumes not fitting in memory.

- Scenario 3: IBiDaPE was set up on a virtualized hardware enviroment and plugged-in to current infrastructure. SQL data was synced in IBiDaPE file system and operations were performed using parallel processing. The building blocks of IBiDaPE were not fine-tuned according to data nature or size.

Table 2 shows the environment setup for each case study scenario. For each scenario, a set of common data manipulation, transformation and computing operations were run triplicate over volumes of $5 \mathrm{~GB}(\approx 25$ million entries $)$, $50 \mathrm{~GB}(\approx 250$ million entries $)$ and $100 \mathrm{~GB}(\approx 0.5$ billion entries) - henceforth also named small, medium and large volumes, respectively - available through standard SQLquery interface. Average and standard deviation were then computed for each test.

Tabela 2. Configuration of test scenarios.

\begin{tabular}{|c|c|}
\hline Scenario & Configuration \\
\hline 1 & $\begin{array}{l}\text { SQL Server (PostgreSQL) } \\
\text { 2-core Intel Xeon CPU @2.66 GHz } \\
8 \text { GB RAM, 500 GB HDD } \\
\text { SQL Client (Commodity Workstation) } \\
\text { 8-core Intel i7 CPU @3.60 GHz } \\
16 \text { GB RAM, 500 GB HDD }\end{array}$ \\
\hline 2 & $\begin{array}{l}\text { Comodity Workstation } \\
\text { 8-core Intel i7 CPU @3.60 GHz } \\
16 \text { GB RAM, 500 GB HDD }\end{array}$ \\
\hline 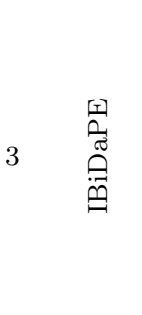 & $\begin{array}{l}\text { Sqoop Server } \\
\text { 4-core Intel Xeon CPU @2.66GHz } \\
8 \text { GB RAM, } 500 \text { GB HDD } \\
\text { 4-node HDFS Cluster } \\
\text { 4-core Intel Xeon CPU @2.66 GHz } \\
8 \text { GB RAM, 500 GB HDD } \\
\text { 5-node Dask Cluster } \\
\text { 6-core Intel Xeon CPU @2.66 GHz } \\
8 \text { GB RAM, 500 GB HDD }\end{array}$ \\
\hline
\end{tabular}

4.3 Results

Table 3 compiles the results for all tests under each considered scenarios and variations of data volumes and operations. Likewise, Figure 3 shows the average execution times (considering all operations) for each scenario. Note that, for the $100 \mathrm{~GB}$ volume, scenarios 1 and 2 did not deliver reasonable execution times or did not completed operations, hence their corresponding points in the curve were roughly extrapolated.

Results showed that the scenario using IBiDaPE (Scenario 3) fared much better for all operations and data volumes, except for the calculations of minimum and maximum values in a column, which seem to be optimized in the DBMS. For small volumes, the adoption of IBiDaPE may not be justifiable as operations execution times for scenarios 1 and 2 are acceptable in the management level. In other hand, the use of IBiDaPE seems to be 
Tabela 3. Comparative of scenarios results.

\begin{tabular}{l|ccc|ccc|ccc}
\hline Scenarios & \multicolumn{3}{|c|}{ Scenario $\mathbf{1}^{1}$} & \multicolumn{3}{c|}{ Scenario 2 } & \multicolumn{3}{c}{ Scenario 3 $^{1}$} \\
Data volumes (GB) & 5 & 50 & 100 & 5 & 50 & 100 & 5 & 50 & 100 \\
\hline Records count & $70[4.5]$ & $1009[16.5]$ & $\mathrm{In}^{2}$ & $211[0.7]$ & $2259[21.8]$ & $\mathrm{In}^{2}$ & $18.0[1.0]$ & $202[2.0]$ & $403[1.7]$ \\
Column values counts & $91[11.5]$ & $1316[11.9]$ & $\mathrm{In}^{2}$ & $451[3.0]$ & $3454[39.8]$ & $\mathrm{In}^{2}$ & $28.3[1.1]$ & $296[15.0]$ & $464[37.1]$ \\
Drop rows with NaNs & $99[34.6]$ & $1327[26.1]$ & $\mathrm{In}^{2}$ & $239[0.2]$ & $2659[36.0]$ & $\mathrm{In}^{2}$ & $22.4[0.4]$ & $249[4.7]$ & $474[7.9]$ \\
Column mean & $90[14.8]$ & $1306[12.1]$ & $\mathrm{In}^{2}$ & $206[0.5]$ & $2194[1.2]$ & $\mathrm{In}^{2}$ & $18.7[0.2]$ & $244[43.1]$ & $414[17.3]$ \\
Column min. value & $0.43[0.1]$ & $50[2.1]$ & $123[3.4]$ & $204[0.1]$ & $2196[1.4]$ & $\mathrm{In}^{2}$ & $18.8[0.1]$ & $241[16.0]$ & $414[5.7]$ \\
Column max. value & $0.47[0.1]$ & $54[3.7]$ & $126[2.7]$ & $205[0.1]$ & $2200[12.3]$ & $\mathrm{In}^{2}$ & $18.5[0.1]$ & $216[6.0]$ & $410[11.6]$ \\
Column sum & $70[1.2]$ & $996[2.4]$ & $\mathrm{In}^{2}$ & $206[0.3]$ & $2387[16.0]$ & $\mathrm{In}^{2}$ & $18.3[0.2]$ & $201[2.0]$ & $411[7.1]$ \\
Column std & $92[14.6]$ & $1314[1.1]$ & $\mathrm{In}^{2}$ & $409[0.2]$ & $4441[13.3]$ & $\mathrm{In}^{2}$ & $18.6[0.3]$ & $219[15.6]$ & $455[23.2]$ \\
Column substring search & $101[11.9]$ & $1408[24.2]$ & $\mathrm{In}^{2}$ & $234[0.5]$ & $2846[53.2]$ & $\mathrm{In}^{2}$ & $18.8[0.4]$ & $221[23.0]$ & $431[6.9]$ \\
Calculate column digest & $149[5.7]$ & $1951[20.3]$ & $\mathrm{In}^{2}$ & $244[0.1]$ & $2619[7.7]$ & $\mathrm{In}^{2}$ & $31.9[0.2]$ & $280[13.1]$ & $467[12.4]$ \\
\hline
\end{tabular}

${ }^{1} \bar{t}(\mathrm{~s})[\sigma(s)]$ - Sample mean and standard deviation for 3 runs expressed in seconds.

${ }^{2}$ In - Infeasible in a reasonable time window (more than one hour).

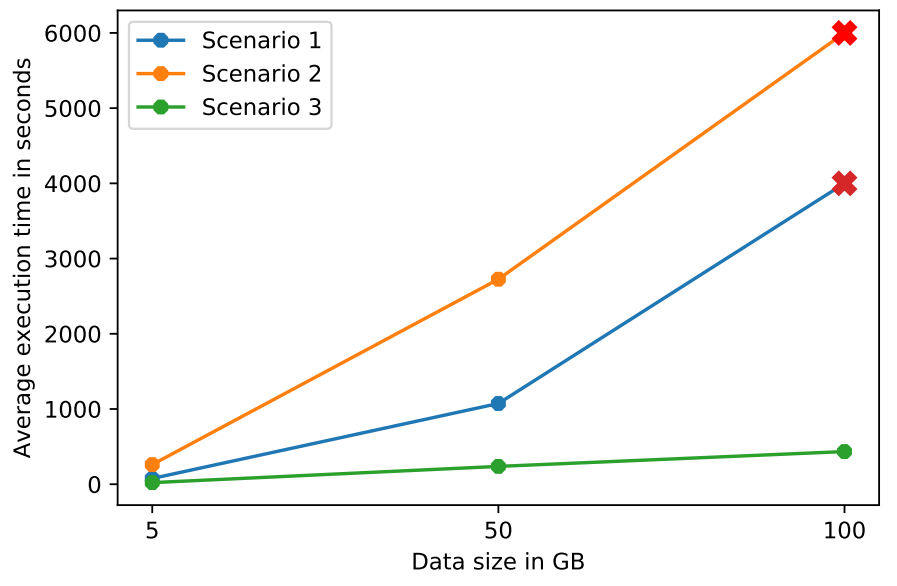

Figura 3. Average execution times for each scenario.

more advantageous as data volumes scales. For medium and large data volumes, reasonable execution times of a few minutes have been noticed when using IBiDaPE while the other test scenarios experienced unsatisfactory execution times from several tens of minutes to some hours. The largest data volume represented a heavy burden for scenarios 1 and 2 since some tests could not complete in a timely manner (notably due to the run triplicate process) or were abruptly interrupted due to memory overloads, random crashes and client timeouts.

\section{CONCLUSION AND FUTURE WORKS}

The proposed solution proved to be effective in providing the capability of timely handling large data volumes to current installed industrial information infrastructure. The approach is meant to be a plug-in to the management layer of traditional industries and may represent a step further in the journey towards the age of Industry 4.0.

It is opportune to clarify that IBiDaPE does not represent or promote an overall structural change to a company. An organization culture change is required to make companies meet the precepts of the advanced manufacture and survive in this new competitive environment. IBiDaPE is supposed to be just a gear in this journey.

IBiDaPE is an early stage work-in-progress plenty of room for improvements in conception, implementation and usage. Work on fine-tuning the building blocks of the solution is a subsequent step towards improving its performance and reliability. Future work also includes comparisons with other Big Data technologies, which can lead to rethinking on the building blocks of proposed solution.

\section{REFERÊNCIAS}

Apache Software Foundation (2019). Apache Sqoop. The Apache Software Foundation. Available at https:// sqoop. apache. org.

Bartodziej, C.J. (2017). The Concept Industry 4.0. Springer Fachmedien Wiesbaden, Berlin. doi:10.1007/ 978-3-658-16502-4.

Basanta-Val, P. (2018). An Efficient Industrial Big-Data Engine. IEEE Transactions on Industrial Informatics, 14(4), 1361-1369. doi:10.1109/TII.2017.2755398.

Brian Hartmann, W.P.K. and Narayanan, S. (2015). Digital manufacturing: The revolution will be virtualized.

Crist, J. (2016). Dask \& Numba: Simple libraries for optimizing scientific python code. Proceedings - 2016 IEEE International Conference on Big Data, Big Data 2016. doi:10.1109/BigData.2016.7840867.

Daniel, J.C. (2018). Data Science at Scale with Python and Dask. Manning Early Access Program. Manning.

Dask Development Team (2016). Dask: Library for dynamic task scheduling. Available at https: // dask. org.

Dean, J. and Ghemawat, S. (2004). MapReduce: Simplified Data Processing on Large Clusters. In Proceedings of the 6th Conference on Symposium on Opearting Systems Design 85 Implementation - Volume 6, OSDI'04. USENIX Association, Berkeley, CA, USA.

Dhruba Borthakur (2019). HDFS Architecture Guide. Apache Software Foundation. Available at https:// hadoop. apache. org/.

GE Intelligent Platforms (2012). The Rise of Industrial Big Data. Technical report, General Electric. Available at http://geautomation. com/download/ rise-industrial-big-data.

Gokalp, M.O., Kayabay, K., Akyol, M.A., Eren, P.E., and Kocyigit, A. (2016). Big Data for Industry 4.0: A Conceptual Framework. In 2016 International Conference on Computational Science and Computational Intelligence (CSCI), 431-434. IEEE. doi:10.1109/CSCI.2016. 0088.

Hajibaba, M. and Gorgin, S. (2014). A review on modern distributed computing paradigms: Cloud computing, jungle computing and fog computing. Journal of 
Computing and Information Technology, 22(2), 69-84. doi:10.2498/cit.1002381.

Jeble, S., Kumari, S., and Patil, Y. (2018). Role of Big Data in Decision Making. Operations and Supply Chain Management: An International Journal, (August), 36. doi:10.31387/oscm0300198.

Kagermann, H., Wahlster, W., and Helbig, J. (2013). Recommendations for implementing the strategic initiative INDUSTRIE 4.0. Technical report, National Academies of Sciences and Engineering.

Lee, J., Bagheri, B., and Kao, H.a. (2015). A CyberPhysical Systems architecture for Industry 4.0-based manufacturing systems. Manufacturing Letters, 3, 1823. doi:10.1016/j.mfglet.2014.12.001.

Lee, J., Kao, H.A., and Yang, S. (2014). Service Innovation and Smart Analytics for Industry 4.0 and Big Data Environment. Procedia CIRP, 16, 3-8. doi:10.1016/j. procir.2014.02.001.

Lu, Y. (2017). Industry 4.0: A survey on technologies, applications and open research issues. Journal of Industrial Information Integration, 6, 1-10. doi:10.1016/j.jii. 2017.04.005.

Mourtzis, D., Vlachou, E., and Milas, N. (2016). Industrial Big Data as a Result of IoT Adoption in Manufacturing. Procedia CIRP, 55. doi:10.1016/j.procir.2016.07.038.

Obitko, M., Jirkovský, V., and Bezdíček, J. (2013). Big Data Challenges in Industrial Automation. In V. Mavrík, J.L.M. Lastra, and P. Skobelev (eds.), Industrial Applications of Holonic and Multi-Agent Systems, 305316. Springer Berlin Heidelberg, Berlin, Heidelberg. doi: 10.1007/978-3-642-40090-2_27.

O'Donovan, P., Leahy, K., Bruton, K., and O'Sullivan, D.T.J. (2015). An industrial big data pipeline for datadriven analytics maintenance applications in large-scale smart manufacturing facilities. Journal of Big Data, 2(1). doi:10.1186/s40537-015-0034-z.

Oussous, A., Benjelloun, F.Z., Ait Lahcen, A., and Belfkih, S. (2017). Big Data technologies: A survey. Journal of King Saud University - Computer and Information Sciences, 30(4). doi:10.1016/j.jksuci.2017.06.001.

Philip Chen, C.L. and Zhang, C.Y. (2014). Data-intensive applications, challenges, techniques and technologies: A survey on Big Data. Information Sciences, 275, 314-347. doi:10.1016/j.ins.2014.01.015.

Shvachko, K., Kuang, H., Radia, S., and Chansler, R. (2010). The hadoop distributed file system. In Proceedings of the 2010 IEEE 26th Symposium on Mass Storage Systems and Technologies (MSST), MSST'10. IEEE Computer Society, Washington, DC, USA. doi: 10.1109/MSST.2010.5496972.

Silva, M., Vieira, E., Signoretti, G., Silva, I., Silva, D., and Ferrari, P. (2018). A Customer Feedback Platform for Vehicle Manufacturing Compliant with Industry 4.0 Vision. Sensors, 18(10). doi:10.3390/s18103298.

Wan, J., Tang, S., Li, D., Wang, S., Liu, C., Abbas, H., and Vasilakos, A.V. (2017). A Manufacturing Big Data Solution for Active Preventive Maintenance. IEEE Transactions on Industrial Informatics, 13(4), 20392047. doi:10.1109/TII.2017.2670505.

Wang, J., Zhang, W., Shi, Y., Duan, S., and Liu, J. (2018). Industrial Big Data Analytics: Challenges, Methodologies, and Applications. CoRR, abs/1807.01016. 